\title{
Catecholaminergic Neuromodulation Shapes Intrinsic MRI Functional Connectivity in the Human Brain
}

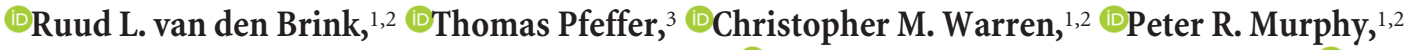 \\ Klodiana-Daphne Tona, ${ }^{1,2}$ Nic J. A. van der Wee, ${ }^{2,4}$ Eric Giltay, ${ }^{4}$ Martijn S. van Noorden, ${ }^{4}$ Serge A.R.B. Rombouts, ${ }^{1,2,5}$

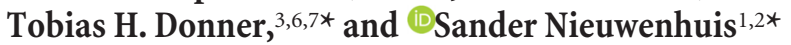 \\ ${ }^{1}$ Institute of Psychology, Leiden University, 2333AK Leiden, The Netherlands, ${ }^{2}$ Leiden Institute for Brain and Cognition, 2333AK Leiden, The Netherlands, \\ ${ }^{3}$ Department of Neurophysiology and Pathophysiology, University Medical Center Hamburg-Eppendorf, 20246, Hamburg, Germany, ${ }^{4}$ Department of \\ Psychiatry and ${ }^{5}$ Department of Radiology, Leiden University Medical Center, Albinusdreef 2, 2333 ZA Leiden, The Netherlands, ${ }^{6}$ Department of Psychology, \\ University of Amsterdam, 1012 WX, Amsterdam, The Netherlands, and ${ }^{7}$ Amsterdam Center for Brain and Cognition, Institute for Interdisciplinary Studies, \\ 1001 NK Amsterdam, The Netherlands
}

The brain commonly exhibits spontaneous (i.e., in the absence of a task) fluctuations in neural activity that are correlated across brain regions. It has been established that the spatial structure, or topography, of these intrinsic correlations is in part determined by the fixed anatomical connectivity between regions. However, it remains unclear which factors dynamically sculpt this topography as a function of brain state. Potential candidate factors are subcortical catecholaminergic neuromodulatory systems, such as the locus ceruleusnorepinephrine system, which send diffuse projections to most parts of the forebrain. Here, we systematically characterized the effects of endogenous central neuromodulation on correlated fluctuations during rest in the human brain. Using a double-blind placebocontrolled crossover design, we pharmacologically increased synaptic catecholamine levels by administering atomoxetine, an NE transporter blocker, and examined the effects on the strength and spatial structure of resting-state MRI functional connectivity. First, atomoxetine reduced the strength of inter-regional correlations across three levels of spatial organization, indicating that catecholamines reduce the strength of functional interactions during rest. Second, this modulatory effect on intrinsic correlations exhibited a substantial degree of spatial specificity: the decrease in functional connectivity showed an anterior-posterior gradient in the cortex, depended on the strength of baseline functional connectivity, and was strongest for connections between regions belonging to distinct resting-state networks. Thus, catecholamines reduce intrinsic correlations in a spatially heterogeneous fashion. We conclude that neuromodulation is an important factor shaping the topography of intrinsic functional connectivity.

Key words: catecholamines; functional connectivity; gain; neuromodulation; norepinephrine; resting-state fMRI

Significance Statement

The human brain shows spontaneous activity that is strongly correlated across brain regions. The factors that dynamically sculpt these inter-regional correlation patterns are poorly understood. Here, we test the hypothesis that they are shaped by the catecholaminergic neuromodulators norepinephrine and dopamine. We pharmacologically increased synaptic catecholamine levels and measured the resulting changes in intrinsic fMRI functional connectivity. At odds with common understanding of catecholamine function, we found (1) overall reduced inter-regional correlations across several levels of spatial organization; and (2) a remarkable spatial specificity of this modulatory effect. Our results identify norepinephrine and dopamine as important factors shaping intrinsic functional connectivity and advance our understanding of catecholamine function in the central nervous system.

\section{Introduction}

The resting-state, here defined as periods during which a participant is not engaged in a complex explicit task, is characterized by fluctuations in neural activity that are correlated across brain regions (Biswal et al., 1995; Leopold et al., 2003; Fox and Raichle, 2007; Hiltunen et al., 2014). Such spontaneous, correlated fluc- 
tuations exhibit a rich spatial (Yeo et al., 2011) and temporal (Allen et al., 2014; Zalesky et al., 2014) structure that is reflective of the brain's functional organization (Tavor et al., 2016). The strength and spatial distribution of these correlated fluctuations are predictive of behavior and pathological conditions (Greicius et al., 2004; De Luca et al., 2005). Moreover, the global structure, or topography, of correlated activity changes dynamically with alterations in conscious state (Barttfeld et al., 2015) and task conditions (Nir et al., 2006; Sepulcre et al., 2010). While the existence and overall spatiotemporal structure of the spontaneous interregional correlations are well established (Fox and Raichle, 2007), uncertainty remains regarding the underlying physiological mechanisms. It has been proposed that correlations across distant brain regions could be induced by brainstem neuromodulatory systems, and in particular the locus ceruleus-norepinephrine (LC-NE) system, which sends diffuse, ascending projections to the forebrain (Leopold et al., 2003; Drew et al., 2008; Schölvinck et al., 2010), where noradrenergic terminals corelease dopamine (DA) (Devoto and Flore, 2006). Here, we examined whether and how the catecholaminergic neuromodulators NE and DA shape correlated fluctuations during rest in the human brain.

A number of observations suggest that catecholamines should generally increase the strength of functional connectivity. Both iontophoretic NE application and DA agonism enhance neuronal responses to excitatory synaptic input (Rogawksi and Aghajanian, 1980; Seamans et al., 2001b; Wang and O'Donnell, 2001). Furthermore, NE and DA can amplify synaptic GABAergic inhibition (Moises et al., 1979; Seamans et al., 2001a). These and other findings have led to the view that catecholamines boost the efficacy of synaptic interactions between neurons (Berridge and Waterhouse, 2003; Winterer and Weinberger, 2004), resulting in an increased difference in firing rates between strongly and weakly active neurons. Such signal amplification yields a systemwide facilitation of signal transmission (Waterhouse et al., 1998). Recent computational work suggests that this effect of catecholamines should boost both positive and negative temporal correlations between the activities of local groups of neurons, resulting in stronger and increasingly clustered network connectivity (Donner and Nieuwenhuis, 2013; Eldar et al., 2013). Putative behavioral and pupillary indices of heightened NE activity have accordingly been shown to co-occur with stronger functional coupling throughout the brain (Eldar et al., 2013). A first consideration of the anatomy of the LC-NE system suggests that these changes in functional connectivity might show little spatial specificity. LC neurons exhibit tightly synchronous firing and collateralize broadly, resulting in largely homogeneous catecholaminergic innervation throughout the brain (Swanson and Hartman, 1975; Aston-Jones et al., 1984; Ishimatsu and Williams, 1996; Berridge and Waterhouse, 2003).

In the present study, we systematically characterized catecholamine effects on the strength and spatial structure of resting-state inter-regional correlations, measured with fMRI. Using a double-blind placebo-controlled crossover design, we manipulated catecholamine activity by administering a single dose of atomoxetine, a selective NE transporter (NET) blocker. Within the cortex, NET is also responsible for DA reuptake, due to the

Correspondence should be addressed to Ruud Lucas van den Brink, Wassenaarseweg 52, 2333AK Leiden, The Netherlands. E-mail: r.l.van.den.brink@fsw.leidenuniv.nl.

DOI:10.1523/JNEUROSCI.0744-16.2016

Copyright $\odot 2016$ the authors $\quad 0270-6474 / 16 / 367866-12 \$ 15.00 / 0$ cortical paucity of DA transporters (Devoto and Flore, 2006). Thus, NET blockers increase both central NE and cortical DA availability (Bymaster et al., 2002; Devoto et al., 2004; Swanson et al., 2006; Koda et al., 2010). We systematically quantified catecholamine effects on functional connectivity: globally, between brain networks, and at the level of individual connections between brain regions. In contrast to the notion of a catecholamineinduced homogeneous increase in functional connectivity, we found that atomoxetine reduced correlations across most pairs of brain regions. Most remarkably, atomoxetine altered the strength of inter-regional correlations in a highly spatially specific manner. These results have important ramifications for our understanding of resting-state activity and central catecholaminergic function.

\section{Materials and Methods}

Participants. Neurologically healthy right-handed individuals $(N=24$, age 19-26 years, 5 male) were recruited and medically screened by a physician for physical health and drug contraindications. Exclusion criteria included: standard contraindications for MRI; current use of psychoactive or cardiovascular medication; a history of psychiatric illness or head trauma; cardiovascular disease; renal failure; hepatic insufficiency; glaucoma; hypertension; drug or alcohol abuse; learning disabilities; poor eyesight (myopia $\leq-6$ diopters); smoking $>5$ cigarettes a day; and pregnancy. All participants gave written informed consent before the experiment and screening, and were compensated with $€ 135$ or course credit.

Design and functional MRI data. We used a double-blind placebocontrolled crossover design. In each of two sessions, scheduled 1 week apart at the same time of day, participants received either a single oral dose of atomoxetine ( $40 \mathrm{mg}$ ) or placebo (125 mg of lactose monohydrate with $1 \%$ magnesium stearate, visually identical to the drug). Elsewhere, we report data showing that the atomoxetine treatment significantly increased salivary levels of cortisol and $\alpha$ amylase, reliable markers of sympathetic nervous system and hypothalamus-pituitary-adrenal axis activation, respectively (C. M. Warren, R. L. van den Brink, S. Nieuwenhuis, and J. A. Bosch, unpublished observations), thus confirming drug uptake. In both sessions, participants were scanned once before pill ingestion $(t=-20 \mathrm{~min})$ and once at $t=90 \mathrm{~min}$, when approximate peak-plasma levels are reached. The interaction contrast (postatomoxetine - preatomoxetine) minus (postplacebo - preplacebo) allowed us to examine the effects of atomoxetine while controlling for other sessionrelated differences. Each scan comprised $8 \mathrm{~min}$ of eyes-open resting-state fMRI. During scanning, the room was dark, and participants fixated on a black fixation cross presented on a gray background.

MRI data collection and preprocessing. All MRI data were collected with a Philips 3T MRI scanner. In each of the scanning sessions, we collected a $\mathrm{T} 2{ }^{*}$-weighted EPI resting-state image (echo time $30 \mathrm{~ms}$, repetition time $2.2 \mathrm{~s}$, flip angle $80^{\circ}$, FOV $80 \times 80 \times 38$ voxels of size $2.75 \mathrm{~mm}$ isotropic, and 216 volumes). To allow magnetic equilibrium to be reached, the first 5 volumes were automatically discarded.

In addition, each time the participant entered the scanner, we collected a $B_{0}$ field inhomogeneity scan (echo time $3.2 \mathrm{~ms}$, repetition time $200 \mathrm{~ms}$, flip angle $30^{\circ}$, and FOV $256 \times 256 \times 80$ voxels with a reconstructed size of $0.86 \times 0.86 \mathrm{~mm}$ with $3-\mathrm{mm}$-thick slices). Finally, at the start of the first session, we collected a high-resolution anatomical T1 image (echo time $4.6 \mathrm{~ms}$, repetition time $9.77 \mathrm{~ms}$, flip angle $8^{\circ}$, and FOV $256 \times 256 \times 140$ voxels with size $0.88 \times 0.88 \mathrm{~mm}$ with 1.2 -mm-thick slices).

We used tools from the FMRIB Software Library for preprocessing of the MRI data (Smith et al., 2004; Jenkinson et al., 2012). EPI scans were first realigned using MCFLIRT motion correction and skull-stripped using BET brain extraction. We used $\mathrm{B}_{0}$ unwarping to control for potential differences in head position each time the participant entered the scanner and resulting differences in geometric distortions in the magnetic field. The $\mathrm{B}_{0}$ scans were first reconstructed into an unwrapped phase angle and magnitude image. The phase image was then converted to units radians per second and median- 
filtered, and the magnitude image was skull-stripped. We then used FEAT to unwarp the EPI images in the $y$-direction with a $10 \%$ signal loss threshold and an effective echo spacing of 0.332656505 .

The unwarped EPI images were then high-pass filtered at $100 \mathrm{~s}$, prewhitened, smoothed at $5 \mathrm{~mm}$ FWHM, and coregistered with the anatomical T1 to $2 \mathrm{~mm}$ isotropic MNI space (degrees of freedom: EPI to T1, 3; T1/EPI to MNI, 12). Any remaining artifacts (e.g., motion residual, susceptibility-motion interaction, cardiac and sinus artifacts) were removed using FMRIB's ICA-based X-noiseifier (Griffanti et al., 2014; Salimi-Khorshidi et al., 2014) with pretrained weights (Standard.RData). Noise classification performance was checked afterward, by manually classifying components as "signal," "noise," or "unknown." Then, the accuracy of the automated artifact detection algorithm was quantified as the percentage of components that had the label "noise" in both classifications. The accuracy was found to be $96.4 \%$ correct. All subsequent analyses were conducted in MATLAB 2012a (The MathWorks).

Physiological recordings and correction. We recorded heart rate using a pulse oximeter and breath rate using a pneumatic belt at $500 \mathrm{~Hz}$ during acquisition of each EPI scan. We used these time series for retrospective image correction (RETROICOR) (Glover et al., 2000). This method assigns cardiac and respiratory phases to each volume in each individual EPI time series, which can then be removed from the data. The physiological time series were first down-sampled to $100 \mathrm{~Hz}$. Next, the pulse oximetry data were bandpass filtered between 0.6 and $2 \mathrm{~Hz}$, and the respiration data were low-pass filtered at $1 \mathrm{~Hz}$, using a two-way FIR filter. We then extracted peaks in each time series corresponding to maximum blood oxygenation and maximum diaphragm expansion. The interpeak intervals were then converted to phase time by linearly interpolating across the intervals to between 0 and $2 \pi$. Next, we used these phase time series to extract the sine- and co-sine components of the dominant and first harmonic Fourier series of each signal. After down-sampling to the EPI sample rate, this yielded 8 regressors (4 cardiac and 4 respiratory) that could then be used to remove cardiac and respiratory effects from the BOLD time series using multiple linear regression. The findings reported here were based on noncorrected data, but we replicated all of our results using the RETROICOR-corrected data (see Results).

Pupillometry. Pupil size was measured from the right eye at $500 \mathrm{~Hz}$ with an MRI-compatible Eyelink 1000 eye tracker. Blinks and other artifacts were interpolated offline using shape-preserving piecewise cubic interpolation. Pupil data were low-pass filtered at $5 \mathrm{~Hz}$ to remove highfrequency noise and $Z$-scored across conditions. Five participants were excluded from pupil-related analyses due to poor signal quality $(>50 \%$ of continuous time series interpolated) or missing data. Of the remaining participants, on average 20\% (SD 9\%) of the data were interpolated.

Brain parcellation and connectivity. Time series of brain regions were extracted for the 90 regions of the Automated Anatomical Labeling (AAL) atlas (Tzourio-Mazoyer et al., 2002) (see Fig. 1a). We did not include the cerebellum because it was not fully inside the FOV for all participants. Following averaging across voxels within each brain region, time series $(M)$ for each run $i$ were $Z$-scored and correlation matrices $(R)$ were computed between them via the following:

$$
R_{i}=\frac{M_{i}^{\prime} \cdot M_{i}}{n T R-1}
$$

where ' denotes transposition and $n T R$ is the number of volumes (211). Because positive and negative correlations jointly determine a network's functional organization (Fox et al., 2005), many prior studies have used the absolute value of the correlation coefficient to describe functional interactions (Achard and Bullmore, 2007; Eldar et al., 2013; Li et al., 2013). Moreover, computational work suggests that catecholamines should boost temporal correlations regardless of their sign (Donner and Nieuwenhuis, 2013; Eldar et al., 2013). We therefore used the absolute correlation coefficient as our measure of connectivity strength. The signed and absolute matrices were very similar because anticorrelations were rare (mean $3.4 \%$ of all connections, SD 3.5\%), as is common when no global signal regression has been performed. In the group- and condition-averaged correlation matrix, $0.28 \%$ were anticorrelations ( 11 of 4005 unique connections; see Fig. 1b). To facilitate comparisons of values across participants, we range-normalized each participant's absolute correlation matrices between 0 and 1 across the 4 conditions. This procedure discarded the between-participant variance while leaving the spatial structure and between-condition variance intact.

In addition, for the postatomoxetine condition time-resolved connectivity (Allen et al., 2014) was computed for 189 tapered windows $w$ of length $n w$ (22 volumes) via the following:

$$
R_{w i}=\frac{M_{w i}^{\prime} \cdot M_{w i}}{n w-1}
$$

The taper was created by convolving a Gaussian (SD 3 TRs) with a rectangle. $R_{w i}$ was Fisher-transformed to stabilize variance across windows. We then again used the absolute value as our measure of connectivity strength. An identical sliding window was applied to the pupil diameter data in the postatomoxetine condition such that for each window in $R_{w i}$ there was a corresponding value of pupil size during that window. Then, we divided up pupil size into 3 equal-sized bins and averaged the corresponding values in $R_{w i}$ for each pupil bin separately. To rule out the possibility that the results depended on the choice of bin size, we also tried alternate bin sizes $(2,5$, and 7 bins) and found similar effects.

Graph-theoretical analysis of global correlation structure. For each condition, we constructed a binary undirected (adjacency) matrix $A$. We did this by first concatenating the correlation matrices across participants such that for each condition we had a brain region by brain region by $N$ $(90 \times 90 \times 24)$ matrix of connectivity. We then assessed with a $t$ test across the participant dimension for each element $y, x$ in the connectivity matrix whether its value differed significantly from the average of its row $y$ or column $x$ (Hipp et al., 2012). In other words, for each connection, we obtained a distribution across participants of weighted values, and two distributions corresponding to the mean weighted values of each brain region that was linked by that particular connection. The connection distribution was then compared with each of the brain region distributions with a $t$ test. If either of the two comparisons was significant, the connection was scored as 1 , and otherwise it was scored as 0 . The $\alpha$ level was set to 0.01 , Bonferroni-corrected for two comparisons to 0.005 (Hipp et al., 2012).

This procedure, as opposed to simply applying a fixed-percentage threshold, results in adjacency matrices that can differ in the number of connections between conditions, and therefore allows the assessment of correlation structure, or degree. We thus quantified the global degree $k$ in each condition as the average across the adjacency matrix (Hipp et al., 2012) via the following:

$$
k=n^{-1} \sum_{x=1}^{n} n^{-1} \sum_{y=1}^{n} A(x, y)
$$

where $n$ is the number of brain regions in the AAL atlas.

To test the prediction that increased catecholamine levels should result in stronger functional connectivity, we used $k$ as our measure of connectivity strength rather than relying on the mean weighted values (i.e., the average of $R_{i}$ ). The binarization of weighted graphs is common in functional network analysis (Achard and Bullmore, 2007; Rubinov and Sporns, 2010; Hipp et al., 2012; Li et al., 2013) and is intended to preserve only the strongest (most probable) connections. This ensures that weak edges, which are more likely to be spurious (Rubinov and Sporns, 2010), do not convolute the global mean. Given that these edges are less likely to reflect true neurophysiological interactions, they are less likely to be sensitive to any experimental manipulation that is specifically intended to alter neurophysiology (in our case, drug intake). Thus, excluding these connections decreases the likelihood of false negatives in betweencondition comparisons of the global mean. In addition, by treating each connection equally (either present or absent), the global mean is not disproportionally influenced by extremely strong connections that are more likely to decrease in strength after an experimental manipulation by virtue of regression toward the mean.

Furthermore, by defining adjacency matrices using a statistical test across participants, each connection that is present in the adjacency matrix is ensured to be reliably expressed across the group of participants for a given condition. Thus, the adjacency matrices are representative of the 
group-level topography of connectivity. We used two measures of clustering, defined using these group-level adjacency matrices, to test the prediction that an increase in central catecholamine levels should be accompanied by more strongly clustered network connectivity. The clustering coefficient $C$ was quantified as the average fraction of triangles $\tau$ around a node, the latter given by the following:

$$
\tau_{x}=2^{-1} \sum A(x, y) A(x, z) A(y, z), \text { where } y, z \in N
$$

and $N$ represents the total set of nodes. $C$ was then given by the following:

$$
C=n^{-1} \sum_{y=1}^{n} n^{-1} \sum_{x=1}^{n} \frac{2 \tau_{x}}{k_{x}\left(k_{x}-1\right)}
$$

The clustering coefficient here is equivalent to the average proportion of the node's neighbors that are in turn neighbors to each other (Watts and Strogatz, 1998; Rubinov and Sporns, 2010). Thus, the clustering coefficient represents the mean fraction of clustering around each node.

Because $C$ is normalized by degree $(k)$ individually per node, it may be biased by nodes with a relatively low $k$. We therefore also included a measure of clustering that is normalized by $k$ collectively and hence does not suffer from the same potential bias. This measure is known as transitivity $(T)$, and is given by the following:

$$
T=\frac{\sum_{x=1}^{n} 2 \tau_{x}}{\sum_{x=1}^{n} k_{x}\left(k_{x}-1\right)}
$$

This is equivalent to the ratio of triangles to triplets in the network. Both clustering coefficient and transitivity capture the extent to which the network is segregated in terms of processing because a large number of triangles implies functional clustering. These two measures were computed using the Brain Connectivity Toolbox (Rubinov and Sporns, 2010). Both clustering and transitivity are (partially) dependent on global degree (van Wijk et al., 2010).

To test statistically whether degree, clustering coefficient and transitivity differed between conditions, we used nonparametric permutation testing. We shuffled the condition labels for each participant before computing the adjacency matrices and then computed the graph-theoretical measures. This was done for 10,000 iterations to produce a null distribution. We then derived a $p$ value for each contrast by dividing the number of null observations less extreme than the observed contrast by the total number of null observations, and subtracting this value from 1 .

Network identification via community detection. We used the Louvain method for community detection optimized for stability (Blondel et al., 2008; Le Martelot and Hankin, 2013) to classify each brain region as belonging to a particular network, or module. This method works by maximizing the number of within-group connections (edges) while minimizing the number of between-group connections via greedy optimization. We first defined an adjacency matrix $A_{s}$ by concatenating the condition-averaged correlation matrices across participants, and then statistically comparing each element $y, x$ to the average of its row $y$ or column $x$, similar as described above. However, to accurately classify networks, we needed to retain only those connections that were most informative about community structure. We therefore promoted sparsity in the condition-averaged adjacency matrix by defining it using a one-tailed $t$ test with a conventional $\alpha$ level $(0.05)$ and a correction for multiple comparisons using the false discovery rate (FDR). This preserved only those connections that were consistently the strongest across participants (16.9\% of all possible connections). We then submitted this sparse condition-averaged adjacency matrix to the Louvain community detection algorithm. The optimization procedure (Le Martelot and Hankin, 2013) ensured a stable solution across multiple runs of the algorithm. In the optimization procedure, the Markov time acts as a resolution parameter that determines the community scale, and thus the number of modules that the algorithm will return. This parameter was set to 0.9 , resulting in 6 separate modules. We set the number of modules to be detected to 6 because, given the relatively coarse anatomical layout of the AAL atlas, this number yielded a relatively reliable modular organi- zation. The community detection and optimization resulted in a "module number" for each AAL brain region indicating to which module it belonged, and a single $Q$ value indicating the strength of modularity.

We first verified whether the $Q$ value was significantly higher than chance. To do so, we generated 10,000 randomized null networks with an identical size, density, and degree distribution as $A_{s}$ (Maslov and Sneppen, 2002), and submitted them to Louvain community detection and optimization to produce a null-distribution of $Q$ values. We then derived a $p$ value for the observed modularity by dividing the number of null $\mathrm{Q}$ values less extreme than the empirical $Q$ value by the total number of null Q values, and subtracting it from 1 .

The observed Q value of 0.46 was significantly higher than chance $(p<0.001)$, showing that group-average connectivity was strongly modular. We then visualized the modular structure by rearranging the condition-averaged correlation matrix by module. The assignment of brain regions to modules corresponded closely to a number of wellcharacterized intrinsic connectivity networks, indicating that the modular structure reflected a functionally meaningful grouping of brain regions.

Graph-theoretical analysis of network structure. The procedure described above allowed us to group brain regions into modules of intrinsically coupled AAL brain regions. We could then use these modules to assess changes in the structure of intrinsic correlations at the within- and between-network level, rather than as a function of the system in its entirety. To do this, we first rearranged the condition-specific adjacency matrices by their module number, and computed average degree of elements within and between modules via the following:

$$
k_{m}=n_{a}^{-1} \sum_{x_{a}=1}^{n_{a}} n_{b}^{-1} \sum_{y_{b}=1}^{n_{b}} A_{s}\left(x_{a}, y_{b}\right)
$$

where $n_{a}$ is the number of brain regions belonging to module $a$ and $n_{b}$ is the number of brain regions in module $b$. This yielded, for each condition, a symmetric and module-by-module matrix of continuous average degree values, in which values on the diagonal indicated the average number of connections within each module, and each value around the diagonal indicated the average number of connections between a combination of modules.

We could then use these "module matrices" to test for atomoxetinerelated changes in degree of the connections within modules, and the connections linking different modules. This allowed us to characterize changes in connectivity in a spatially more specific way than for global degree. We again used nonparametric permutation testing, similar as described for global degree, except that it was done for individual elements within the module matrices.

Control analyses using an alternate atlas and multiple thresholds. To rule out the possibility that our results were specific to the use of the AAL atlas, we repeated all of our key analyses using the atlas made available by Craddock et al. (2012), which comprised 87 distinct regions after excluding the cerebellum, and found similar effects in terms of both direction and significance. Moreover, to verify that our results were independent of the statistical threshold used to define the adjacency matrices, we conducted a control analysis in which a range of adjacency matrices was created per condition with varying condition-averaged connection densities (40\%-75\%). This was done by progressively raising/lowering the $\alpha$ level of the $t$ test that was used to determine whether a connection is present or absent (see above). Then, for each threshold we computed the graph-theoretical measures, and for each condition and measure separately calculated the area under the curve across thresholds. This allowed us to compare the area under the curve between conditions with permutation testing (10,000 iterations). For all measures, the critical interaction contrast was significant and in the same direction as our original findings (see Results).

Controlling for regression toward the mean. The correlation between baseline coupling strength and the atomoxetine-related change in coupling strength (see Fig. $3 e$; see Results) is confounded by regression toward the mean. That is, if two particular brain regions show strong baseline coupling, then simply by chance they are more likely to show a reduction under atomoxetine, and so a negative correlation is likely to occur. We therefore controlled for regression toward the mean using 


\section{a}

Automated Aanatomical Labeling (AAL) atlas
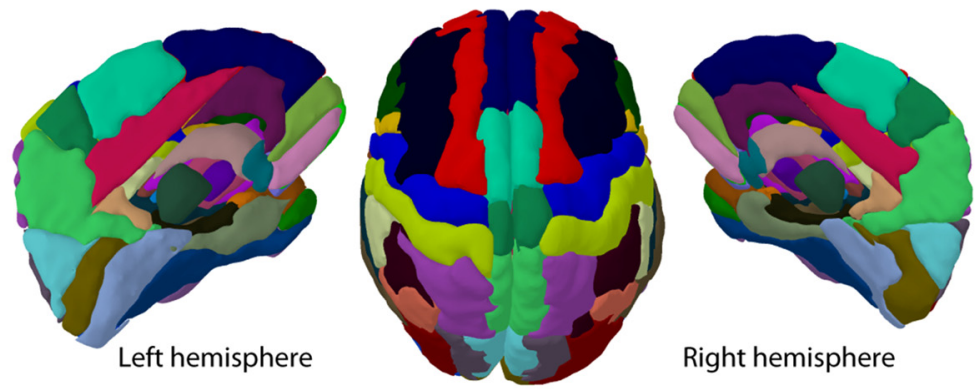

Right hemisphere b

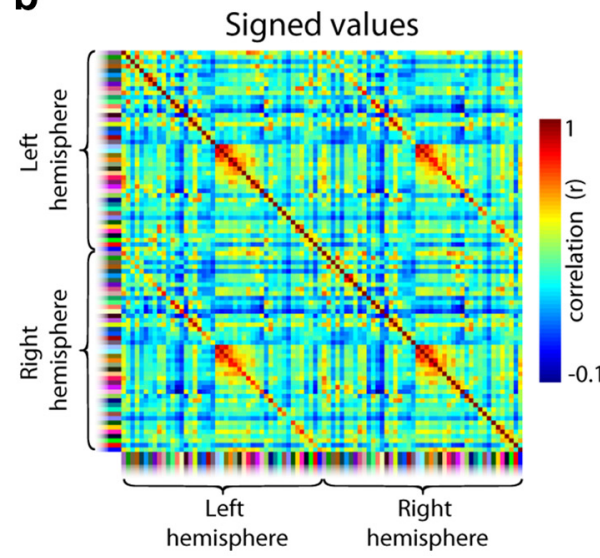

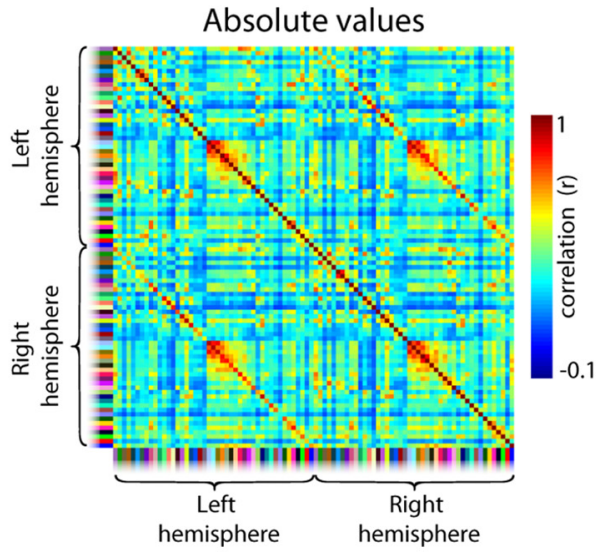

C
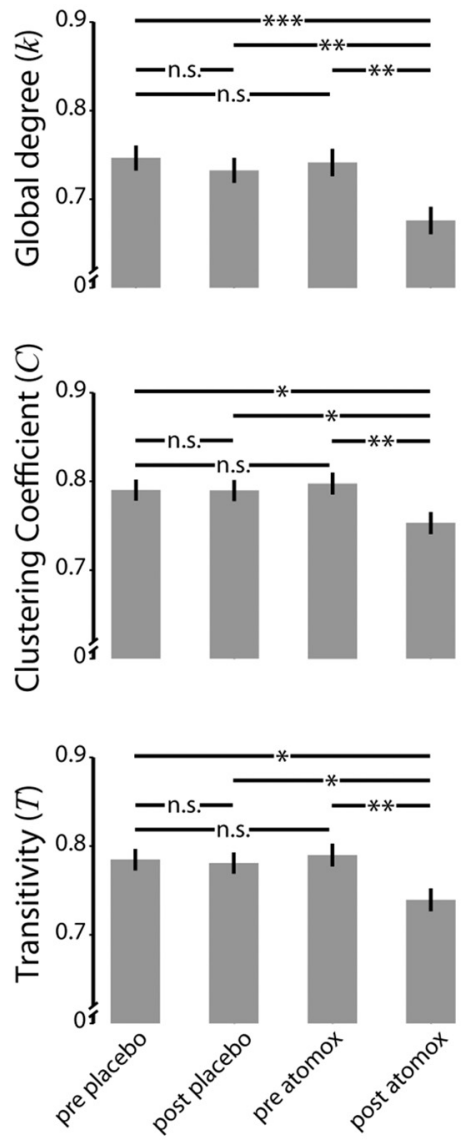

Figure 1. Inter-regional correlation and global graph-theoretical results. $\boldsymbol{a}$, Topography of the AAL atlas. Each brain region within hemispheres has a unique color. $\boldsymbol{b}$, Condition-averaged inter-regional correlation. Both the signed and absolute values are shown. Color labels on the left and bottom axes represent brain regions in $\boldsymbol{a}$. $\boldsymbol{c}$, Atomoxetine-related effects on global graph-theoretical measures. Error bars indicate the SD of the bootstrapped null-distribution. n.s., Not significant. ${ }^{*} p<0.05$. $^{* *} p<0.01$. ${ }^{* * *} p<0.001$.

permutation testing. For 10,000 permutations, we shuffled the condition labels across participants before computing the atomoxetine-related change in coupling strength. We then computed the correlation between baseline coupling and atomoxetine-related change in coupling to produce a distribution of correlation coefficients under the null hypothesis of regression toward the mean. Finally, we derived a $p$ value for the empirical correlation coefficient by dividing the number of null observations less extreme than the correlation coefficient by the total number of null observations, and subtracting this value from 1 . This $p$ value indicated the significance of the observed correlation coefficient beyond regression toward the mean.

Analysis of BOLD signal variance. We calculated for each participant and each AAL brain region the fractional amplitude (i.e., variance) of low-frequency fluctuations in the non- $Z$-scored BOLD time series (fALFF) (Zou et al., 2008). This measure indexes the relative contribution of low-frequency $(0.01-0.08 \mathrm{~Hz})$ fluctuations to the total amplitude spectrum. We compared fALFF between conditions using repeatedmeasures ANOVA. Additionally, for each participant, we correlated the atomoxetine-related change in fALFF with the atomoxetine-related change in inter-regional correlation strength across AAL brain regions. We then compared the distribution of Fisher-transformed correlation coefficients to zero using a two-tailed $t$ test. Very similar results were obtained using alternative measures of variance (e.g., average $0.01-0.08$ $\mathrm{Hz}$ amplitude or the signal SD rather than fractional amplitude).

\section{Results}

Atomoxetine reduces global degree and clustering

In a first set of analyses, we examined the effect of atomoxetine on graph-theoretical summary measures of functional connectivity strength. We parcellated each participant's brain into 90 separate regions according to the AAL atlas (Tzourio-Mazoyer et al., 2002) (Fig. 1a) and computed the correlation between the $Z$-scored time series of all pairs of regions (Fig. $1 b$ ). We then took the absolute correlation coefficient as our measure of functional connectivity strength (see Materials and Methods). In general, functional connectivity was strongest between visual cortical areas and between homolog areas in both hemispheres (Fig. $1 b$ ), consistent with a host of previous work (Fox and Raichle, 2007).

For each condition (preplacebo, postplacebo, preatomoxetine, postatomoxetine), we constructed a binary matrix of connections (edges) between pairs of brain regions that consistently differed in strength across participants from the average of other connections involving either of the two brain regions (following Hipp et al., 2012). Graph theory allowed us to capture different properties of these matrices of intrinsic correlations in a small number of diagnostic scalar quantities (Bullmore and Sporns, 2009; Rubinov and Sporns, 2010). Specifically, we assessed three such measures: the global degree, which indexes the number of strongly correlated regions (above a certain threshold; see Materials and Methods) in the network, and two descriptors of the extent to which network connectivity is clustered in segregated local groups of brain regions: clustering coefficient and transitivity, both of which are (partially) dependent on the strength of connectivity (van Wijk et al., 2010; Eldar et al., 2013). If cat- 

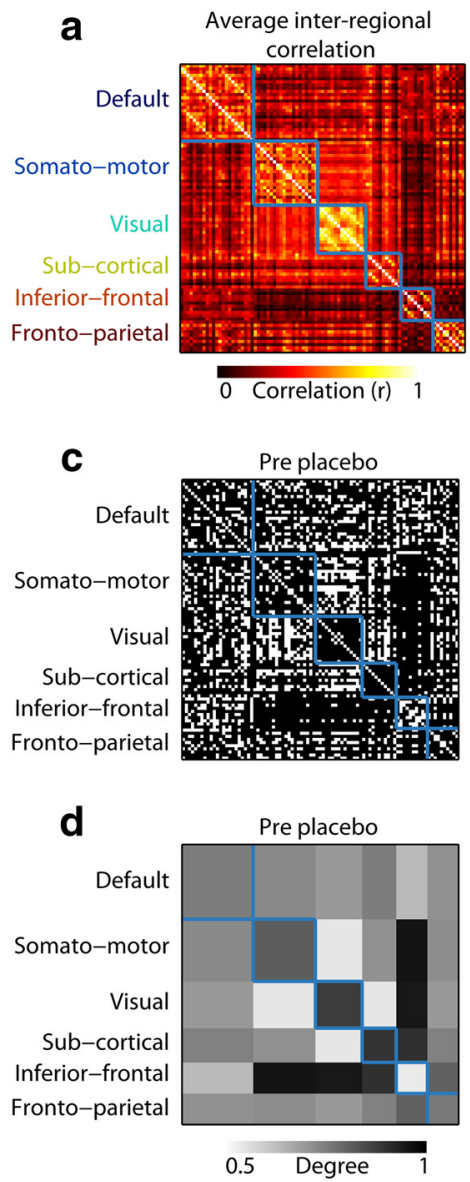

b
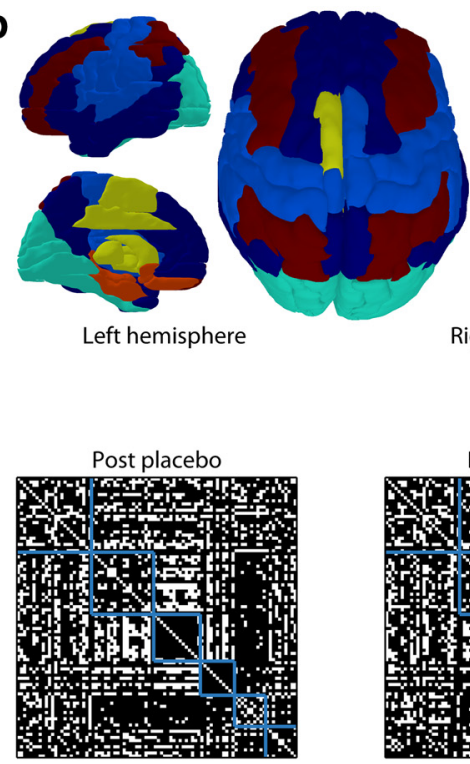

Post placebo

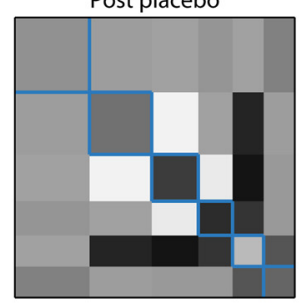

Right hemisphere

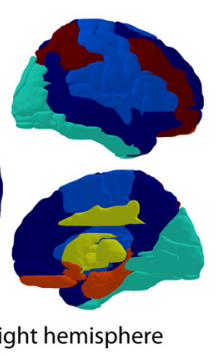

Pre atomox

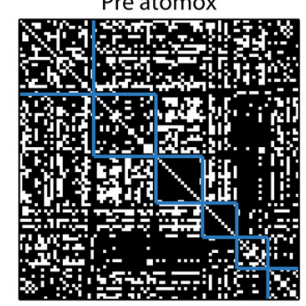

Pre atomox

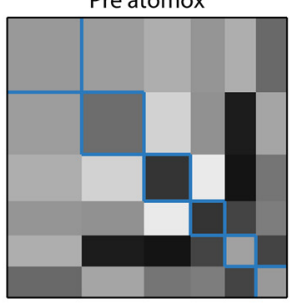

e

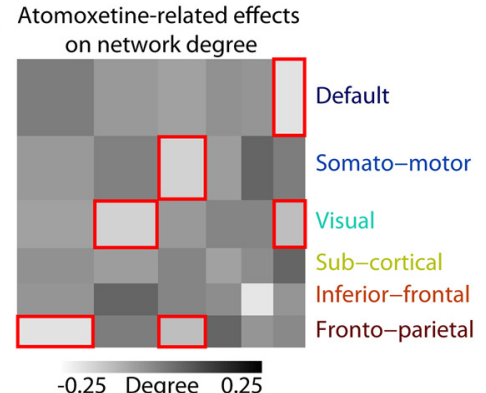

-0.25 Degree 0.25

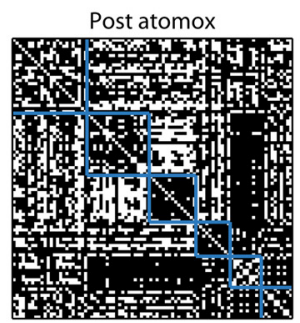

Post atomox

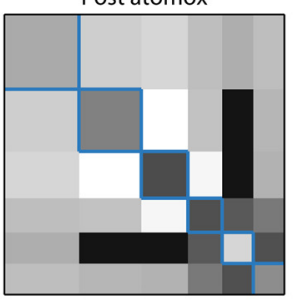

Figure 2. Intrinsic connectivity networks and changes in graph-theoretical measures of network structure. $\boldsymbol{a}$, Condition-averaged inter-regional correlation arranged by network. The networks are outlined in blue. $\boldsymbol{b}$, Topography of functional networks. Colors correspond to the labels in $\boldsymbol{a}$. $\boldsymbol{c}$, Condition-specific adjacency matrices arranged by network. Black elements indicate that a connection is present. $\boldsymbol{d}$, Average degree for within- and between-network connections. To facilitate visual comparison, the size of each network is the same as in $\boldsymbol{c}$. However, all statistical comparisons were conducted on symmetrically sized matrices in which each network contributed equally to the global mean. $e$, Atomoxetine-induced changes in degree for connections within and between networks. Shades of gray represent the value of the interaction contrast (postatomoxetine - preatomoxetine) minus (postplacebo - preplacebo). Significant ( $p<0.05$ ) changes in degree are outlined in red.

echolamines increase global functional connectivity, then atomoxetine should increase all three measures.

Figure $1 c$ shows that atomoxetine significantly reduced the number of strong correlations present in the network, as indicated by lower global degree. This was reflected in a significant interaction between treatment and time $(p=0.039)$. A similar pattern of results was found for the two measures of clustering, both of which decreased in magnitude (Fig. 1c): clustering coefficient $(p=0.043)$ and transitivity $(p=0.048)$. Thus, atomoxetine reduced the number of strongly correlated brain regions, as well as the extent to which correlated brain regions formed local functional ensembles. Together, these results show that atomoxetine decreases, rather than increases, overall inter-regional correlations in the brain at rest.

\section{Atomoxetine reduces internetwork degree}

Many studies of resting-state activity in humans have revealed a consistent set of groups, or modules, of brain regions that are characterized by strong coupling between brain regions belonging to the same module, and weaker coupling between brain regions belonging to different modules (Bullmore and Sporns, 2009). These modules are often referred to as "intrinsic functional connectivity networks" (Fox and Raichle, 2007). In a next set of analyses, we investigated atomoxetine-related changes in the strength of functional connectivity within and between these networks.

To do this, we arranged the connectivity matrix by network (Fig. 2a) (Blondel et al., 2008). This resulted in 6 functional networks that correspond closely to previously reported restingstate networks (Yeo et al., 2011; Zalesky et al., 2014). Based on their topography (Fig. 2b), we termed them: default; somatomotor; visual; subcortical; inferior-frontal; and frontoparietal networks. We then calculated the average number of connections within and between these networks, resulting in a $6 \times 6$ network degree matrix for each condition (Fig. 2c,d). Finally, we examined atomoxetinerelated changes in within- and between-network degree using permutation testing. This allowed us to explore whether changes in functional connectivity occurred in intranetwork or internetwork connections. The atomoxetine-related reduction in global degree (Fig. 1c) is visible in the network degree matrices as an overall increase in "brightness" in Figure $2 d$ (right).

Consistent with the decrease in global degree reported above, we observed only atomoxetine-related reductions in network degree (Fig. 2e). The interaction between treatment and time was significant for the connections between the visual and somatomotor networks $(p<0.001)$, between the visual and frontoparietal networks $(p=0.044)$, and between the frontoparietal and default networks $(p<0.001)$. After using the FDR $(q=0.05)$ to 
a Atomoxetine-related effect on inter-regional correlation Signed values
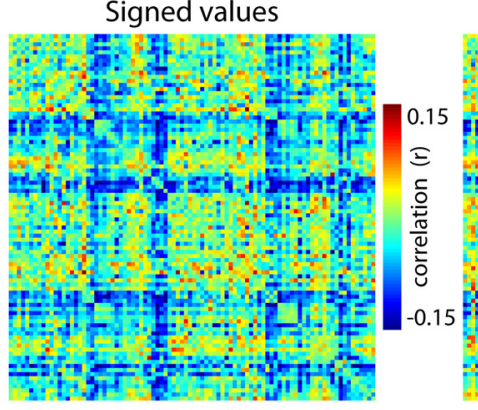

Absolute values

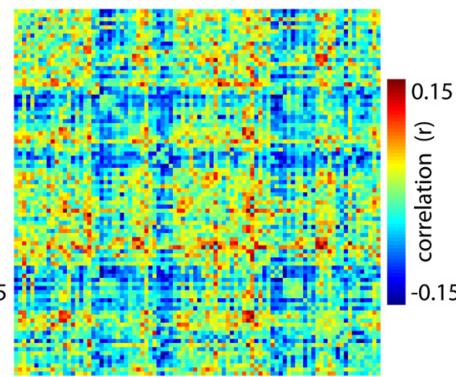

b

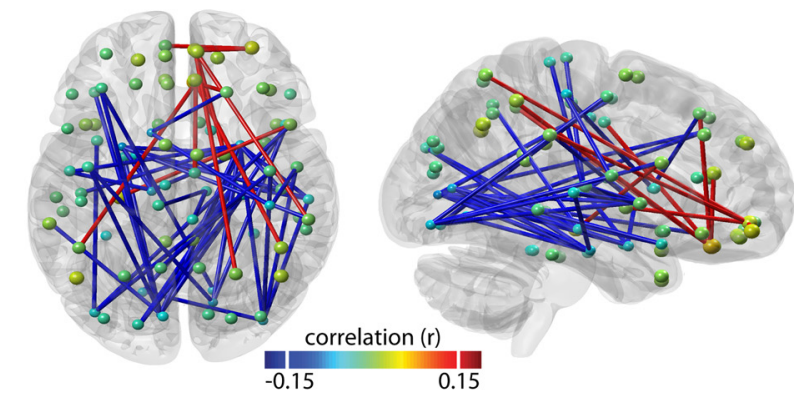

C

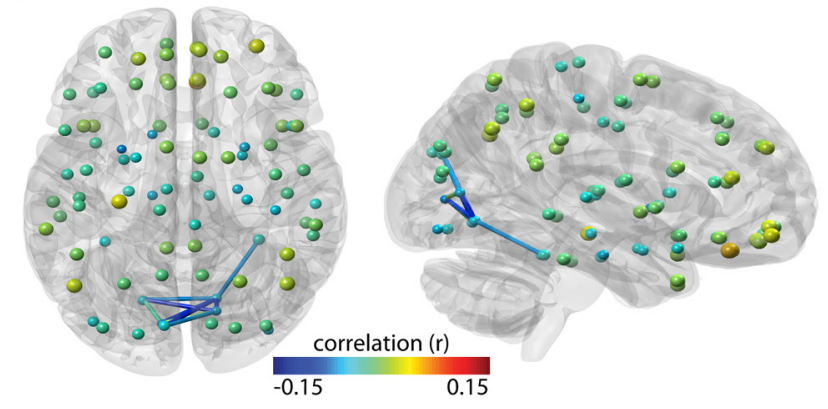

d

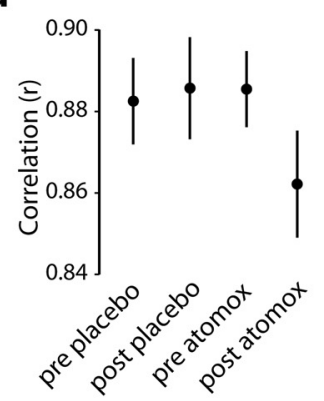

e

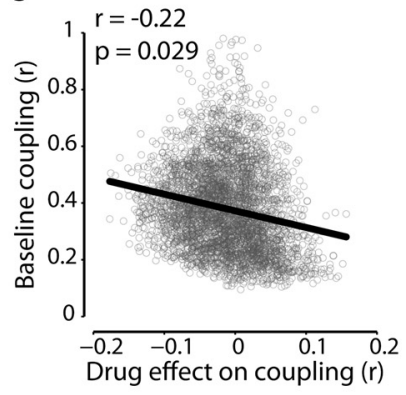

Figure 3. Atomoxetine-related effects on inter-regional correlation. $\boldsymbol{a}$, Region-by-region matrix of atomoxetine-related changes in inter-regional correlation strength. Colors represent the value of the interaction contrast (postatomoxetine - preatomoxetine) minus (postplacebo - preplacebo). Blue represents reduced correlation following atomoxetine. The matrices are organized following Figure $1 b . \boldsymbol{b}$, Atomoxetine-related effect on the absolute inter-regional correlation coefficient, rendered in 3D with an arbitrary threshold applied. White dashes in the color bar indicate threshold. Spheres are placed in the center of mass of their respective AAL atlas regions. Both the size and color indicate the average atomoxetine-related effect on coupling (i.e., the average across rows or columns in $\boldsymbol{a}$ ). $\boldsymbol{c}$, Transverse (top is anterior) and sagittal (right is anterior) view on 3D rendering of significant correlation changes, resulting from the whole-brain two-step analysis. Individual connections that changed significantly with atomoxetine are plotted as cylinders between the corresponding regions. $\boldsymbol{d}$, Inter-regional correlation in each condition, averaged across the significant connections shown in c. Error bars indicate the SEM. e, Correlation between baseline inter-regional correlation strength (collapsed across preplacebo and preatomoxetine) and change with atomoxetine. Each dot represents a unique region-by-region connection. Self-connections were excluded.

correct for multiple comparisons, all connections, except the connection between the visual and frontoparietal networks, remained significant. However, when comparing the area under the curve across a range of thresholds (see Materials and Methods), all connections remained significant after FDR correction.

Interestingly, all significant reductions in network degree were in connections between (as opposed to within) functional networks. Thus, the most robust decreases in functional coupling occurred for connections linking functionally dissociable groups of brain regions. These results corroborate the conclusion drawn above: that atomoxetine decreased inter-regional correlations in the brain at rest. More importantly, these results provide a first indication that this reduction in inter-regional correlations is not spatially homogeneous across the brain. In the following, we further characterize the spatial heterogeneity of the atomoxetineinduced reductions in inter-regional correlations.

\section{Regionally specific reductions and baseline-dependent changes in connectivity with atomoxetine}

Having assessed the topographical changes induced by atomoxetine at the global level and the level of functional networks, we next assessed differences in the strength of inter-regional correlations at the level of individual connections between brain regions, using the absolute correlation coefficient. We found that atomoxetine altered correlation strength in a strikingly structured fashion (Fig. 3a): In general, connectivity was reduced by atomoxetine, especially in posterior brain regions (Fig. $3 b$ ). These observations align with our findings of reductions in internet- work degree involving the visual system. To quantify these effects, we used a two-step procedure. Specifically, we first derived a set of data-driven hypotheses by identifying, in the first half of the fMRI volumes, the limited number of individual connections that exhibited an atomoxetine-related change in connectivity that was reliable across participants $(p<0.05$, using a two-tailed $t$ test), thereby reducing the number of comparisons for the subsequent step. We then retested those connections using the (independent) second half of the volumes and selected those that again showed a systematic atomoxetine-related change in correlation strength ( $p<0.005$, two-tailed). Atomoxetine significantly lowered correlation strength in a cluster of occipital brain regions (Fig. $3 c, d$ ), specifically correlations between left calcarine cortex and right calcarine cortex/bilateral lingual gyrus; between left cuneus and right calcarine cortex/lingual gyrus; between left lingual gyrus and right calcarine cortex/lingual gyrus; and between right lingual gyrus and right calcarine cortex/right fusiform gyrus. These contiguous connections remained significant after applying a highly conservative cluster size threshold $(p<$ 0.0001 ), obtained by generating a distribution of maximum cluster sizes under the null hypothesis with permutation testing (Nichols and Holmes, 2002). Thus, the cluster involved significantly more connections than would be expected by chance.

We did not find significant changes in connectivity between structures of the basal ganglia, which have been widely studied in relation to catecholaminergic drug effects (Sulzer et al., 2016). This lack of an atomoxetine-related effect in the human basal ganglia is consistent with the observation that the basal ganglia 
a Condition-averaged correlation seeded from visual cortex

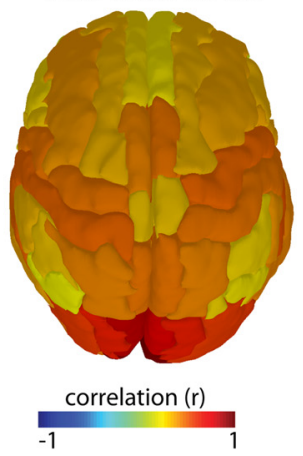

b

b

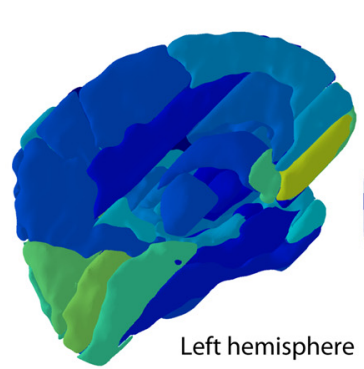

Left hemisphere

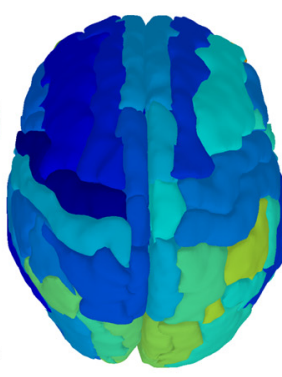

correlation $(r)$

$-0.15 \quad 0.15$
Atomoxetine-related effect on inter-regional correlation seeded from visual cortex

Figure 4. Atomoxetine reduces correlation strength between early visual (pericalcarine) cortex and the rest of the brain. $\boldsymbol{a}$, Topography of condition-averaged correlation seeded from left and right early visual cortex. $\boldsymbol{b}$, Topography of atomoxetine-related effects on correlation seeded from left and right early visual cortex. Colors represent the value of the interaction (postatomoxetine preatomoxetine) minus (postplacebo - preplacebo). c, Median correlation values across the brain seeded from left and right early visual cortex. Error bars indicate the SEM. receive relatively sparse noradrenergic innervation (Aston-Jones et al., 1984), and with findings that atomoxetine has little effect on DA levels within the basal ganglia of rodents (Bymaster et al., 2002). Indeed, unlike in the cortex, in the basal ganglia there is an abundance of DA transporter (Sulzer et al., 2016), so DA reuptake is not dependent on the NE transporter. Thus, our finding that atomoxetine reduced the strength of inter-regional correlations in (predominantly visual) cortical areas is consistent with the specific effect of atomoxetine on synaptic catecholamine levels within the cortex.

In sum, atomoxetine lowered the strength of correlations between visual cortical brain regions (Fig. 3c), regions that on average showed strong connectivity (Figs. $1 b, 2 a$ ). This raises the question whether the atomoxetine-induced change in connectivity was dependent on the baseline level of connectivity between any pair of brain regions. To address this question, we correlated weighted coupling strength collapsed across the preplacebo and preatomoxetine conditions (i.e., baseline connectivity) with the coupling change following atomoxetine (controlling for regression toward the mean with permutation testing). The observed correlation between baseline connectivity strength and the change with atomoxetine was significant $(r=-0.22, p=0.029$; Fig. $3 e$ ). This indicates that the strongest functional connections tended to show the largest connectivity reductions after atomoxetine, and vice versa.

\section{Atomoxetine induces decoupling of early visual cortex from the rest of the brain}

As noted above, atomoxetine reduced correlations between occipital brain regions. To establish whether these occipital regions also showed reduced coupling to the rest of the brain, as suggested by visual inspection (Fig. 3b), we computed a summary statistic (median) of correlation strength between early visual (pericalcarine) cortex and all other AAL atlas regions. There were no differences between left and right early visual cortex, so we collapsed the data across hemispheres.

Average connectivity seeded from early visual cortex is shown in Figure $4 a$. Atomoxetine reduced connectivity between early visual cortex and the rest of the brain, as reflected by a significant interaction between treatment and time $\left(F_{(1,23)}=5.31, p=\right.$ 0.031; Fig. $4 b, c)$. The only significant pairwise comparison was postatomoxetine versus preatomoxetine $\left(t_{(23)}=2.34, p=0.028\right)$. Together, these results suggest that the early visual cortical areas not only decoupled from each other following atomoxetine (Fig. $3 c$ ) but also from the rest of the brain.

The results of our analyses at the level of individual connections between brain regions converge with those at the global level and at the level of networks of brain regions, showing that atomoxetine decreased functional connectivity. In addition, the results show that atomoxetine modulated functional connectivity in a highly regionally specific fashion, with more robust changes in visual cortex than in other brain areas.

\section{Excluding alternative explanations}

In five sets of control analyses, we ruled out the possibility that the atomoxetine-related changes in inter-regional correlations were driven either by local changes in BOLD variance, by retinal effects due to pupil dilation associated with atomoxetine, by head motion, by saccade-related retinal transients, or by atomoxetineinduced changes in physiology (heart rate and breath rate). First, the correlation coefficient between two signals is their covariance normalized by the signals' variances. Thus, it is possible that the observed changes in inter-regional correlations are caused by local changes in variance alone (Haynes et al., 2005; Freeman et al., 2011), rather than by changes in covariance (i.e., the degree to which the BOLD signals in two regions fluctuated together). If this is the case, then the atomoxetine-related change in average inter-regional correlation and the atomoxetine-related change in BOLD signal variance should be negatively correlated across brain regions. Instead, we found a positive relationship between changes in inter-regional correlation and changes in BOLD variance, which was consistent across participants $\left(t_{(23)}=3.36, p=\right.$ 0.003 ; Fig. 5), ruling out variance as a confound. Moreover, there was no interaction between treatment and time in overall BOLD variance $\left(F_{(1,23)}=0.71, p=0.40\right)$, or in variance for only the occipital brain regions that showed reduced atomoxetine-related inter-regional correlation $\left(F_{(1,23)}=0.41, p=0.53\right)$.

Second, because atomoxetine increased the size of the pupil (Fig. 6a), it is conceivable that this peripheral effect, rather than the effect of atomoxetine on central catecholamine levels, was driving the changes in inter-regional correlation in visual cortex (Haynes et al., 2004). To examine this potential confound, we binned inter-regional correlation by pupil size in the postatomoxetine condition, focusing on those correlations that showed a significant reduction under atomoxetine. If larger pupil size is responsible for the reduction in correlations, then time periods 


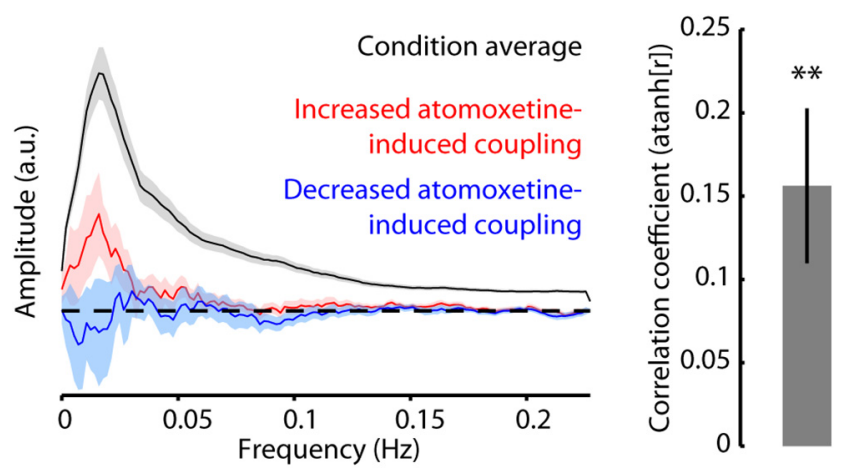

Figure 5. Spectral BOLD characteristics and the relation with inter-regional correlations. Left, Atomoxetine-induced changes in spectral amplitude for AAL brain regions that showed an atomoxetine-induced increase (red) and decrease (blue) in inter-regional correlation strength. Brain region and condition-averaged amplitude areas shown in black. Right, Mean correlation between the region-averaged atomoxetine-induced change in coupling strength and fractional amplitude of low-frequency BOLD fluctuations. Error bars indicate the SEM. ${ }^{* *} p<0.01$.

during which the pupil is large should be associated with weaker correlations than time periods during which the pupil is small. Interestingly, we found the opposite pattern (Fig. 6b,c): stronger correlations for large pupil $\left(t_{(18)}=2,84, p=0.010\right)$, ruling out an interpretation in terms of pupil size.

Third, head motion can have a strong influence on the strength of inter-regional correlations (Van Dijk et al., 2012). To rule out the possibility that our key finding of atomoxetinerelated changes in inter-regional correlation was driven by head motion, we first compared head motion between conditions. Neither mean head motion nor mean absolute head motion differed between conditions (all $p$ values $>0.05$ ). No participant's head motion exceeded $2 \mathrm{~mm}$, indicating that overall there was little head motion. However, general mild head motion tends to increase correlations between proximate areas and decrease connectivity between distant areas (Van Dijk et al., 2012). Thus, head motion can potentially lead to spatially heterogeneous effects on connectivity in a manner that is related to the distance between brain areas. To rule out the possibility that the spatial structure of atomoxetine-related changes in connectivity was driven by subtle (nonsignificant) differences in head motion between conditions, we correlated Euclidean distance between the center of mass of each pair of AAL brain areas and the strength of functional connectivity between those areas, for each participant and each condition. We then compared the distribution of Fisher-transformed correlation coefficients between conditions. If head motion is responsible for the observed change in connectivity between conditions, then the correlation between Euclidean distance and strength of connectivity should also differ between conditions. However, we did not find any differences between conditions (all $p$ values $>0.05)$, ruling out head motion as a confound.

Fourth, it is possible that that the atomoxetine-related reduction in the strength of correlation between visual cortical areas occurred due to differences between conditions in saccaderelated retinal transients. To rule out this possibility, we extracted several eye movement metrics from the eye tracker gaze position data using the EYE-EEG toolbox (Dimigen et al., 2011). There was no interaction between treatment and time for any of the metrics: the number of saccades $\left(F_{(1,18)}=0.47, p=0.50\right)$, median saccade amplitude $\left(F_{(1,18)}=0.45, p=0.51\right)$, median saccade duration $\left(F_{(1,18)}=0.11, p=0.74\right)$, or median saccade peak velocity $\left(F_{(1,18)}=3.32, p=0.085\right)$. This latter trend was driven by a numeric difference between the preplacebo and postplacebo conditions. Preatomoxetine and postatomoxetine did not differ significantly in saccade peak velocity $\left(t_{(18)}=-0.43, p=0.67\right)$. Together, these results show that our key result of an atomoxetine-related reduction in the strength of correlation between visual cortical regions was unlikely to be driven by saccaderelated retinal transients.

Finally, atomoxetine significantly increased breath rate $\left(F_{(1,23)}=8.96, p=0.007\right)$ and heart rate $\left(F_{(1,23)}=4.66, p=\right.$ $0.041)$, as reflected by a significant interactions between treatment and time. We therefore corrected the BOLD time series using the RETROICOR method (see Materials and Methods) (Glover et al., 2000). The average $R^{2}$ of the physiology regressors was relatively low (0.034), indicating that physiology accounted for a small proportion of the total BOLD variance (which was likely the result of artifact removal by FMRIB's ICA-based X-noiseifier) (Griffanti et al., 2014; Salimi-Khorshidi et al., 2014). Nevertheless, to conclusively rule out atomoxetine-related changes in physiology as confounds, we repeated the key analyses on the physiology-corrected data. All three global graphtheoretical measures remained significant and in the same direction as reported above (all $p$ values $<0.05$ ). We also found significant reductions in network degree in the same internetwork connections (all $p$ values $<0.05$ ). Last, we observed a similar contiguous cluster of significantly reduced inter-regional correlations within visual cortex (all $p$ values $<0.005$, and clustercorrected at $p<0.0001)$. Thus, our key results were unlikely to be driven by atomoxetine-related changes in physiology.

\section{Discussion}

Using a pharmacological manipulation, we examined the effects of increased extracellular levels of the catecholamines NE and DA on resting-state fMRI connectivity in the human brain. First, we found that our manipulation reduced the strength of interregional correlations across three levels of spatial organization, indicating that catecholamines reduce the strength of functional interactions during rest. Second, this modulatory effect on the structure of resting-state correlations exhibited a substantial degree of spatial specificity, indicating that catecholamines differentially reduce spontaneous correlations between select brain regions. These two key findings are surprising in light of the common understanding of the neurophysiology and computational function of catecholaminergic systems. They also identify catecholaminergic neuromodulation as an important factor shaping the spatial structure and strength of intrinsic functional connectivity in the human brain.

Our first key finding is that atomoxetine, a selective NET blocker that increases synaptic NE and DA levels (Bymaster et al., 2002; Devoto et al., 2004; Invernizzi and Garattini, 2004; Swanson et al., 2006; Koda et al., 2010), reduced the strength of interregional correlations. Specifically, atomoxetine reduced the strength of connectivity globally (Fig. 1c), between nodes belonging to distinct intrinsic connectivity networks (Fig. 2e), and between individual brain regions within the visual system (Fig. 3c). This consistent pattern of results seems to be at odds with the notion of a facilitative effect of catecholamines on brain-wide signal transmission (Aston-Jones and Cohen, 2005; Eldar et al., 2013). One possible explanation for this discrepancy lies in the fact that, in our experiment, participants did not actively respond to incoming sensory information. According to a recent theory, the effects of NE on neural activity strongly depend on interactions with local glutamate release (Mather et al., 2015). Accordingly, enhanced NE may have qualitatively different effects during task processing, associated with relatively high glutamate 
activity, than during states of relative cortical quintessence (i.e., at rest), associated with relatively low glutamate activity. In line with this possibility, Coull et al. (1999) showed dissociable effects of the $\alpha 2$ adrenergic agonist clonidine on positron emission tomography effective connectivity obtained during task performance and during rest. Whereas during task performance clonidine increased connectivity between frontal and parietal cortical regions, during rest clonidine reduced connectivity from frontal cortex to thalamus, and in connections to and from visual cortex. Two other studies that used NE drugs also provided evidence for regional reductions in connectivity strength during rest (McCabe and Mishor, 2011; Metzger et al., 2015). These studies, however, only used a small number of seed regions to assess connectivity, and hence did not examine large-scale topographical changes.

Our second key finding is that atomoxetine resulted in spatially heterogeneous changes in inter-regional correlations. For example, atomoxetine caused a reduction in the number of strongly correlated brain regions between (but not within) distinct resting-state networks (Fig. 2e). Furthermore, the effect of atomoxetine on inter-regional correlations was dependent on the baseline level of coupling: the strongest functional connections tended to show the largest connectivity reductions after atomoxetine (Fig. $3 d$ ). How can such spatially structured effects of catecholamines come about? First, recent anatomical tracing work has suggested that the projection profile of the LC is more heterogeneous than once thought (Schwarz and Luo, 2015). For example, even though on the whole there is broad collateralization within the LC-NE system, subpopulations of LC neurons selectively innervate distinct brain regions (Chandler et al., 2014; Schwarz et al., 2015). Moreover, subpopulations of LC neurons that differ in their afferent projection profile also show marked differences in their firing characteristics (Chandler et al., 2014). The firing modes of LC neurons in turn have differentiable effects on neuronal synchronization within the cortex (Safaai et al., 2015). Importantly, LC neurons have been reported to corelease DA (Devoto and Flore, 2006). Thus, spatially selective effects of catecholamines on correlated fluctuations in the brain can be achieved via a heterogeneous cortical innervation by the LC.

Second, heterogeneity in the effect of catecholamines on interregional correlations could be achieved by regional differences in the expression of different receptor types. For example, expression of the $\alpha 2$ receptor approximately follows an anterior to posterior gradient (Nahimi et al., 2015), with particularly strong expression in primary visual cortex (Zilles and Amunts, 2009). Interestingly, we observed an anterior to posterior gradient in the effect of atomoxetine on the strength of correlations (Fig. 3b). Moreover, we found a pronounced reduction in the strength of correlations between regions within visual cortex, and between early visual cortex and the rest of the brain (Fig. 4). The similarity between the spatial distributions of $\alpha 2$ receptors and the effects of atomoxetine thus warrants further investigation into the relationship between specific NE receptor types and their influence on correlated activity across the brain.

A number of limitations of the present study should be acknowledged. First, we examined the effects of only one dose $(40 \mathrm{mg})$ of atomoxetine. Dose-dependent pharmacological effects of catecho-
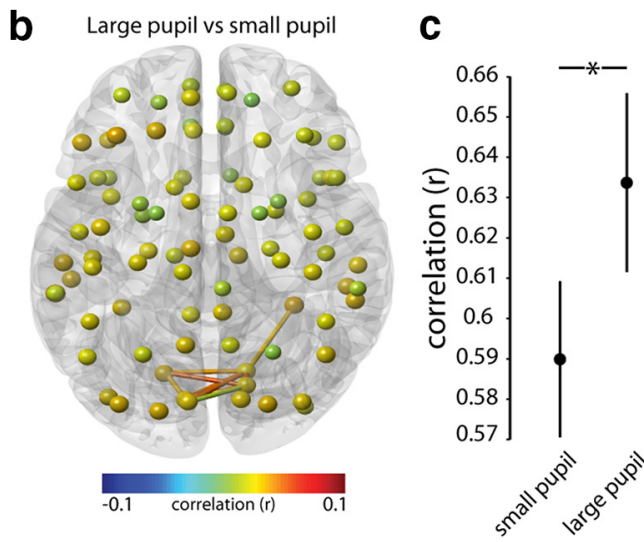

Figure 6. $\boldsymbol{a}$, Atomoxetine effect on pupil diameter. $\boldsymbol{b}, \boldsymbol{c}$, Correlation strength in the postatomoxetine condition binned by pupil size, only for connections that showed an atomoxetine-related reduction in inter-regional correlation. Error bars indicate the SEM. n.s., Not significant. ${ }^{*} p<0.05$. ${ }^{* *} p<0.001$.

laminergic drugs on neural function are not uncommon (Berridge and Waterhouse, 2003). Future work on the neurochemical basis of functional connectivity will need to examine dose-dependent effects of atomoxetine, and other catecholaminergic drugs, with different pharmacokinetic profiles. Second, we do not know whether atomoxetine would have similar effects on functional connectivity in clinical populations characterized by disturbed catecholaminergic function (e.g., attention deficit hyperactivity disorder and depression). Third, although we used BOLD activity as a proxy for neural activity, the link between neuronal interactions and BOLD activity is not entirely clear (Logothetis, 2008). Models of catecholamine function make predictions about how NE and DA should affect neural communication. However, the translation of these predictions to BOLD correlations is not straightforward. Last, we used an atlasbased brain parcellation to investigate inter-regional correlations. Thus, the spatial resolution of our analyses was restricted by the resolution of the atlas. Future work, using voxel-level approaches, is needed to investigate more fine-grained spatial effects of catecholamine levels on functional connectivity.

The synaptic effects of catecholamines have been relatively well charted (Berridge and Waterhouse, 2003; Winterer and Weinberger, 2004). However, there is considerable uncertainty about how these low-level effects translate to system-wide functional interactions. Recently, Safaai et al. (2015) provided an important first glimpse into how the LC-NE system modulates spontaneous cortical activity and how this modulation in turn affects sensory processing in anesthetized rats. Specifically, they showed that LC bursts can both attenuate and enhance processing of sensory stimuli depending on their timing relative to the stimulus and the cortical activity state. However, the effects of catecholamines on the large-scale communication between distant brain areas and their neurophysiological underpinnings remain exceedingly unexplored. Our finding that atomoxetine reduced inter-regional correlations in a spatially structured manner thus calls for novel work on the neural mechanisms that produce such effects.

Theory and evidence indicate that the topography of intrinsic fMRI correlations is dictated to an important extent by the fixed anatomical connectivity of each brain region (Deco et al., 2011, 2013). That is, brain regions that are anatomically strongly connected are more likely to show strong functional coupling than those that are connected weakly or only indirectly. However, within the constraints of physical connectivity, there is substantial room for state-dependent movement in functional topologi- 
cal space (Allen et al., 2014; Zalesky et al., 2014; Barttfeld et al., 2015). Our results identify NE and DA as important factors driving these movements, and thus suggest that spontaneous fluctuations of catecholamine levels can serve to flexibly alter the structure of spontaneous correlations both globally and in specific brain regions, around the anatomical backbone.

\section{References}

Achard S, Bullmore E (2007) Efficiency and cost of economical brain functional networks. PLoS Comput Biol 3:e17. CrossRef Medline

Allen EA, Damaraju E, Plis SM, Erhardt EB, Eichele T, Calhoun VD (2014) Tracking whole-brain connectivity dynamics in the resting state. Cereb Cortex 24:663-676. CrossRef Medline

Aston-Jones G, Cohen JD (2005) An integrative theory of locus coeruleusnorepinephrine function: adaptive gain and optimal performance. Annu Rev Neurosci 28:403-450. CrossRef Medline

Aston-Jones G, Foote SL, Bloom FE (1984) Anatomy and physiology of locus coeruleus neurons: functional implications. In: Norepinephrine (Ziegler MG, Lake CR, eds), pp 92-116. Baltimore: Williams and Wilkins.

Barttfeld P, Uhrig L, Sitt JD, Sigman M, Jarraya B, Dehaene S (2015) Signature of consciousness in the dynamics of resting-state brain activity. Proc Natl Acad Sci U S A 112:887-892. CrossRef Medline

Berridge CW, Waterhouse BD (2003) The locus coeruleus-noradrenergic system: modulation of behavioral state and state-dependent cognitive processes. Brain Res Rev 42:33-84. CrossRef Medline

Biswal B, Yetkin FZ, Haughton VM, Hyde JS (1995) Functional connectivity in the motor cortex of resting human brain using echo-planar MRI. Magn Reson Med 34:537-541. CrossRef Medline

Blondel VD, Guillaume JL, Lambiotte R, Lefebvre E (2008) Fast unfolding of communities in large networks. J Stat Mech 10:P10008.

Bullmore E, Sporns O (2009) Complex brain networks: graph theoretical analysis of structural and functional systems. Nat Rev Neurosci 10: 186-198. CrossRef Medline

Bymaster FP, Katner JS, Nelson DL, Hemrick-Luecke SK, Threlkeld PG, Heiligenstein JH, Morin SM, Gehlert DR, Perry KW (2002) Atomoxetine increases extracellular levels of norepinephrine and dopamine in prefrontal cortex of rat: a potential mechanism for efficacy in attention deficit/hyperactivity disorder. Neuropsychopharmacology 27:699-711. CrossRef Medline

Chandler DJ, Gao WJ, Waterhouse BD (2014) Heterogeneous organization of the locus coeruleus projections to prefrontal and motor cortices. Proc Natl Acad Sci U S A 111:6816-6821. CrossRef Medline

Coull JT, Büchel C, Friston KJ, Frith CD (1999) Noradrenergically mediated plasticity in a human attentional neuronal network. Neuroimage 10: 705-715. CrossRef Medline

Craddock RC, James GA, Holtzheimer PE 3rd, Hu XP, Mayberg HS (2012) A whole brain fMRI atlas generated via spatially constrained spectral clustering. Hum Brain Mapp 33:1914-1928. CrossRef Medline

Deco G, Jirsa VK, McIntosh AR (2011) Emerging concepts for the dynamical organization of resting-state activity in the brain. Nat Rev Neurosci 12:43-56. CrossRef Medline

Deco G, Ponce-Alvarez A, Mantini D, Romani GL, Hagmann P, Corbetta M (2013) Resting-state functional connectivity emerges from structurally and dynamically shaped slow linear fluctuations. J Neurosci 33:1123911252. CrossRef Medline

De Luca M, Smith S, De Stefano N, Federico A, Matthews PM (2005) Blood oxygenation level dependent contrast resting state networks are relevant to functional activity in the neocortical sensorimotor system. Exp Brain Res 167:587-594. CrossRef Medline

Devoto P, Flore G (2006) On the origin of cortical dopamine: is it a cotransmitter in noradrenergic neurons? Curr Neuropharmacol 4:115-125. CrossRef Medline

Devoto P, Flore G, Pira L, Longu G, Gessa GL (2004) Alpha2-adrenoceptor mediated co-release of dopamine and noradrenaline from noradrenergic neurons in the cerebral cortex. J Neurochem 88:1003-1009. CrossRef Medline

Dimigen O, Sommer W, Hohlfeld A, Jacobs AM, Kliegl R (2011) Coregistration of eye movements and EEG in natural reading: analyses and review. J Exp Psychol Gen 140:552-572. CrossRef Medline

Donner TH, Nieuwenhuis S (2013) Brain-wide gain modulation: the rich get richer. Nat Neurosci 16:989-990. CrossRef Medline
Drew PJ, Duyn JH, Golanov E, Kleinfeld D (2008) Finding coherence in spontaneous oscillations. Nat Neurosci 11:991-993. CrossRef Medline

Eldar E, Cohen JD, Niv Y (2013) The effects of neural gain on attention and learning. Nat Neurosci 16:1146-1153. CrossRef Medline

Fox MD, Raichle ME (2007) Spontaneous fluctuations in brain activity observed with functional magnetic resonance imaging. Nat Rev Neurosci 8:700-711. CrossRef Medline

Fox MD, Snyder AZ, Vincent JL, Corbetta M, Van Essen DC, Raichle ME (2005) The human brain is intrinsically organized into dynamic, anticorrelated functional networks. Proc Natl Acad Sci U S A 102:96739678. CrossRef Medline

Freeman J, Donner TH, Heeger DJ (2011) Inter-area correlations in the ventral visual pathway reflect feature integration. J Vis 11:pii15. CrossRef Medline

Glover GH, Li TQ, Ress D (2000) Image-based method for retrospective correction of physiological motion effects in fMRI: RETROICOR. Magn Reson Med 44:162-167. CrossRef Medline

Greicius MD, Srivastava G, Reiss AL, Menon V (2004) Default-mode network activity distinguishes Alzheimer's disease from healthy aging: evidence from functional MRI. Proc Natl Acad Sci U S A 101:46374642. CrossRef Medline

Griffanti L, Salimi-Khorshidi G, Beckmann CF, Auerbach EJ, Douaud G, Sexton CE, Zsoldos E, Ebmeier KP, Filippini N, Mackay CE, Moeller S, Xu J, Yacoub E, Baselli G, Ugurbil K, Miller KL, Smith SM (2014) ICA-based artefact removal and accelerated fMRI acquisition for improved resting state network imaging. Neuroimage 95:232-247. CrossRef Medline

Haynes JD, Lotto RB, Rees G (2004) Responses of human visual cortex to uniform surfaces. Proc Natl Acad Sci U S A 101:4286-4291. CrossRef Medline

Haynes JD, Driver J, Rees G (2005) Visibility reflects dynamic changes of effective connectivity between V1 and fusiform cortex. Neuron 46 : 811-821. CrossRef Medline

Hiltunen T, Kantola J, Abou Elseoud A, Lepola P, Suominen K, Starck T, Nikkinen J, Remes J, Tervonen O, Palva S, Kiviniemi V, Palva JM (2014) Infra-slow EEG fluctuations are correlated with resting-state network dynamics in fMRI. J Neurosci 34:356-362. CrossRef Medline

Hipp JF, Hawellek DJ, Corbetta M, Siegel M, Engel AK (2012) Large-scale cortical correlation structure of spontaneous oscillatory activity. Nat Neurosci 15:884-890. CrossRef Medline

Invernizzi RW, Garattini S (2004) Role of presynaptic alpha2-adrenoceptors in antidepressant action: recent findings from microdialysis studies. Prog Neuropsychopharmacol Biol Psychiatry 28:819-827. CrossRef Medline

Ishimatsu M, Williams JT (1996) Synchronous activity in locus coeruleus results from dendritic interactions in pericoerulear regions. J Neurosci 16:5196-5204. Medline

Jenkinson M, Beckmann CF, Behrens TE, Woolrich MW, Smith SM (2012) Fsl. Neuroimage 62:782-790. CrossRef Medline

Koda K, Ago Y, Cong Y, Kita Y, Takuma K, Matsuda T (2010) Effects of acute and chronic administration of atomoxetine and methylphenidate on extracellular levels of noradrenaline, dopamine and serotonin in the prefrontal cortex and striatum of mice. J Neurochem 114:259-270. CrossRef Medline

Le Martelot E, Hankin C (2013) Multi-scale community detection using stability optimisation. Int J Web Based Communities 9:323-348. CrossRef

Leopold DA, Murayama Y, Logothetis NK (2003) Very slow activity fluctuations in monkey visual cortex: implications for functional brain imaging. Cereb Cortex 13:422-433. CrossRef Medline

Li N, Ma N, Liu Y, He XS, Sun DL, Fu XM, Zhang X, Han S, Zhang DR (2013) Resting-state functional connectivity predicts impulsivity in economic decision-making. J Neurosci 33:4886-4895. CrossRef Medline

Logothetis NK (2008) What we can do and what we cannot do with fMRI. Nature 453:869-878. CrossRef Medline

Maslov S, Sneppen K (2002) Specificity and stability in topology of protein networks. Science 296:910-913. CrossRef Medline

Mather M, Clewett D, Sakaki M, Harley CW (2015) Norepinephrine ignites local hot spots of neuronal excitation: how arousal amplifies selectivity in perception and memory. Behav Brain Sci 1-100.

McCabe C, Mishor Z (2011) Antidepressant medications reduce subcortical-cortical resting-state functional connectivity in healthy volunteers. Neuroimage 57:1317-1323. CrossRef Medline

Metzger CD, Wiegers M, Walter M, Abler B, Graf H (2015) Local and global 
resting state activity in the noradrenergic and dopaminergic pathway modulated by reboxetine and amisulpride in healthy subjects. Int J Neuropsychopharmacol 19:piipyv080. CrossRef Medline

Moises HC, Woodward DJ, Hoffer BJ, Freedman R (1979) Interactions of norepinephrine with Purkinje cell responses to putative amino acid neurotransmitters applied by microiontophoresis. Exp Neurol 64:493-515. CrossRef Medline

Nahimi A, Jakobsen S, Munk OL, Vang K, Phan JA, Rodell A, Gjedde A (2015) Mapping alpha2 adrenoceptors of the human brain with 11Cyohimbine. J Nucl Med 56:392-398. CrossRef Medline

Nichols TE, Holmes AP (2002) Nonparametric permutation tests for functional neuroimaging: a primer with examples. Hum Brain Mapp 15:1-25. Medline

Nir Y, Hasson U, Levy I, Yeshurun Y, Malach R (2006) Widespread functional connectivity and fMRI fluctuations in human visual cortex in the absence of visual stimulation. Neuroimage 30:1313-1324. CrossRef Medline

Rogawski MA, Aghajanian GK (1980) Modulation of lateral geniculate neurone excitability by noradrenaline microiontophoresis or locus ceuruleus stimulation. Nature 287:731-734. CrossRef Medline

Rubinov M, Sporns O (2010) Complex network measures of brain connectivity: uses and interpretations. Neuroimage 52:1059-1069. CrossRef Medline

Safaai H, Neves R, Eschenko O, Logothetis NK, Panzeri S (2015) Modeling the effect of locus coeruleus firing on cortical state dynamics and singletrial sensory processing. Proc Natl Acad Sci U S A 112:12834-12839. CrossRef Medline

Salimi-Khorshidi G, Douaud G, Beckmann CF, Glasser MF, Griffanti L, Smith SM (2014) Automatic denoising of functional MRI data: combining independent component analysis and hierarchical fusion of classifiers. Neuroimage 90:449-468. CrossRef Medline

Schölvinck ML, Maier A, Ye FQ, Duyn JH, Leopold DA (2010) Neural basis of global resting-state fMRI activity. Proc Natl Acad Sci U S A 107:1023810243. CrossRef Medline

Schwarz LA, Luo L (2015) Organization of the locus coeruleus-norepinephrine system. Curr Biol 25:R1051-R1056. CrossRef Medline

Schwarz LA, Miyamichi K, Gao XJ, Beier KT, Weissbourd B, DeLoach KE, Ren J, Ibanes S, Malenka RC, Kremer EJ, Luo L (2015) Viral-genetic tracing of the input-output organization of a central noradrenaline circuit. Nature 524:88-92. CrossRef Medline

Seamans JK, Gorelova N, Durstewitz D, Yang CR (2001a) Bidirectional dopamine modulation of GABAergic inhibition in prefrontal cortical pyramidal neurons. J Neurosci 21:3628-3638. Medline

Seamans JK, Durstewitz D, Christie BR, Stevens CF, Sejnowski TJ (2001b) Dopamine D1/D5 receptor modulation of excitatory synaptic inputs to layer V prefrontal cortex neurons. Proc Natl Acad Sci U S A 98:301-306. CrossRef Medline

Sepulcre J, Liu H, Talukdar T, Martincorena I, Yeo BT, Buckner RL (2010) The organization of local and distant functional connectivity in the human brain. PLoS Comput Biol 6:e1000808. CrossRef Medline

Smith SM, Jenkinson M, Woolrich MW, Beckmann CF, Behrens TE, Johansen-Berg H, Bannister PR, De Luca M, Drobnjak I, Flitney DE, Niazy RK, Saunders J, Vickers J, Zhang Y, De Stefano N, Brady JM, Mat- thews PM (2004) Advances in functional and structural MR image analysis and implementation as FSL. Neuroimage 23 [Suppl 1]:S208-S219.

Sulzer D, Cragg SJ, Rice ME (2016) Striatal dopamine neurotransmission: regulation of release and uptake. Basal Ganglia 6:123-148. CrossRef Medline

Swanson CJ, Perry KW, Koch-Krueger S, Katner J, Svensson KA, Bymaster FP (2006) Effect of the attention deficit/hyperactivity disorder drug atomoxetine on extracellular concentrations of norepinephrine and dopamine in several brain regions of the rat. Neuropharmacology 50:755-760. CrossRef Medline

Swanson LW, Hartman BK (1975) The central adrenergic system: an immunofluorescence study of the location of cell bodies and their efferent connections in the rat utilizing dopamine-beta-hydroxylase as a marker. J Comp Neurol 163:467-505. CrossRef Medline

Tavor I, Parker Jones O, Mars RB, Smith SM, Behrens TE, Jbabdi S (2016) Task-free MRI predicts individual differences in brain activity during task performance. Science 352:216-220. CrossRef Medline

Tzourio-Mazoyer N, Landeau B, Papathanassiou D, Crivello F, Etard O, Delcroix N, Mazoyer B, Joliot M (2002) Automated anatomical labeling of activations in SPM using a macroscopic anatomical parcellation of the MNI MRI single-subject brain. Neuroimage 15:273-289. CrossRef Medline

Van Dijk KR, Sabuncu MR, Buckner RL (2012) The influence of head motion on intrinsic functional connectivity MRI. Neuroimage 59:431-438. CrossRef Medline

van Wijk BC, Stam CJ, Daffertshofer A (2010) Comparing brain networks of different size and connectivity density using graph theory. PLoS One 5:e13701. CrossRef Medline

Wang J, O'Donnell P (2001) D1 dopamine receptors potentiate NMDAmediated excitability increase in layer $\mathrm{V}$ prefrontal cortical pyramidal neurons. Cereb Cortex 11:452-462. CrossRef Medline

Waterhouse BD, Moises HC, Woodward DJ (1998) Phasic activation of the locus coeruleus enhances responses of primary sensory cortical neurons to peripheral receptive field stimulation. Brain Res 790:33-44. CrossRef Medline

Watts DJ, Strogatz SH (1998) Collective dynamics of 'small-world' networks. Nature 393:440-442. CrossRef Medline

Winterer G, Weinberger DR (2004) Genes, dopamine and cortical signalto-noise ratio in schizophrenia. Trends Neurosci 27:683-690. CrossRef Medline

Yeo BT, Krienen FM, Sepulcre J, Sabuncu MR, Lashkari D, Hollinshead M, Roffman JL, Smoller JW, Zöllei L, Polimeni JR, Fischl B, Liu H, Buckner RL (2011) The organization of the human cerebral cortex estimated by intrinsic functional connectivity. J Neurophysiol 106:1125-1165. CrossRef Medline

Zalesky A, Fornito A, Cocchi L, Gollo LL, Breakspear M (2014) Timeresolved resting-state brain networks. Proc Natl Acad Sci U S A 111: 10341-10346. CrossRef Medline

Zilles K, Amunts K (2009) Receptor mapping: architecture of the human cerebral cortex. Curr Opin Neurol 22:331-339. CrossRef Medline

Zou QH, Zhu CZ, Yang Y, Zuo XN, Long XY, Cao QJ, Wang YF, Zang YF (2008) An improved approach to detection of amplitude of lowfrequency fluctuation (ALFF) for resting-state fMRI: fractional ALFF. J Neurosci Methods 172:137-141. CrossRef Medline 